\title{
Polyurethane Synthesis from Sugarcane Bagasse Organosolv and Kraft Lignin
}

\author{
Chularat Sakdaronnarong $^{1, a^{*}}$, Nattawee Srimarut ${ }^{1}$ \\ and Navadol Laosiripojana ${ }^{2, b}$ \\ ${ }^{1}$ Department of Chemical Engineering, Faculty of Engineering, Mahidol University, Putthamonthon, \\ Nakorn Pathom 73170 Thailand \\ ${ }^{2}$ The Joint Graduate School of Energy and Environment (JGSEE), King Mongkut's University of \\ Technology Thonburi, Tungkru, Bangkok 10140 Thailand \\ achularat.sak@mahidol.ac.th, ${ }^{b}$ navadol@jgsee.kmutt.ac.th
}

Keywords: Sugarcane bagasse; Organosolv lignin; Liquefied Kraft lignin; Rigid polyurethane synthesis

\begin{abstract}
Sugarcane bagasse (SCB) was subjected to a single-step fractionation and hydrolysis reaction in the presence of various organic solvents. The reaction was performed at $170{ }^{\circ} \mathrm{C}$ for $3 \mathrm{~h}$ when sodium hydroxide was used as catalyst. The results showed that suitable solvents significantly enhanced the yield of lignin fractionation and simultaneous hydrolysis reaction took place indicated by an increase of hydroxyl groups in lignin molecules. Lignin-based polyurethane (LPU) from SCB organosolv lignin polyols had relatively better mechanical and thermal resistant properties compared to LPU from liquefied Kraft lignin from pulp and paper industry.
\end{abstract}

\section{Introduction}

Polyurethanes (PUs) are polymers containing urethane linkages in molecules. PUs are synthesized by the exothermic reactions of alcohols having two or more reactive hydroxyl (-OH) groups per molecule i.e. diols, triols, polyols and isocyanates having more than one reactive isocyanate group (-NCO) per molecule i.e. diisocyanates and polyisocyanates. Demands of rigid PUs have been increasing for insulation in refrigeration appliances, boardstock and sandwich panels. Therefore, the properties required are low density, low thermal conductivity, low moisture permeability, high dimensional stability, good adhesive properties and low cost [1]. However, concerns have been raised for the depletion of petroleum resources which are precursors for polyols production. Utilization of industrial waste to replace polyols from fossil resource for PU production along with the optimization of process energy and operating cost have been intensively investigated.

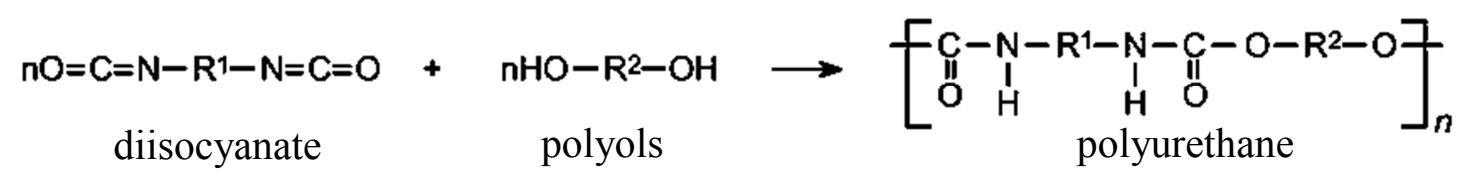

Fig.1 Polyurethane synthesis

Lignocellulosic biomass is the most abundant renewable resource in Thailand found in the form of agricultural and agro-industrial residues such as sugarcane bagasse, rice straw, oil palm shrank. Valorization of cellulose and hemicellulose which are sources of polysaccharides via chemical and biotechnological conversion to various building block chemicals, biofuels, and bio-based material has been intensively developed [2, 3]. In contrast, lignin valorization technologies are considerably less developed. Due to the complex and heterogeneous structure along with the presence of various linkages between the monomers, the conversion of lignin to value-added chemicals is more substantially difficult than carbohydrates. Lignin monomers are composed of coniferyl alcohol, coumaryl alcohol and sinapyl alcohol [4], so that it has potential to substitute petroleum-based polyols as it contains aliphatic and phenolic hydroxyl groups, therefore it is feasibly utilized to incorporate in polyurethane synthesis [5]. Replacing polyols derived from petroleum with polyols from renewable resources can have a great impact on the polyurethane industry.

Nevertheless, large molecules of native lignin and small amount of $\mathrm{OH}$ group found in lignin molecules are problems for polymerization of LPU as it requires $\mathrm{OH}$ groups to react with 
isocyanate groups. To develop the process for enhancement of hydroxyl groups within lignin molecules, this work focused on investigation of lignin fractionation and hydrolysis in aqueous organosolv system in the presence of base catalyst. The energy-efficient lignin fractionation process has been proposed to apply for cellulosic ethanol production in which polysaccharides are separated for ethanol production and lignin in black liquor is able to use for lignin-based polyurethane production.

\section{Experimental}

Sugarcane bagasse lignin fractionation

SCB was subjected to aqueous organosolv fractionation system in the presence of $\mathrm{NaOH}$ catalysts. The reaction was carried out at $170{ }^{\circ} \mathrm{C}$ for $3 \mathrm{~h}$. The organic solvents included ethanolamine, ethylene glycol, amyl alcohol, ethanol, glycerol, butanol and dimethylformamide. After reaction, solid fraction which was cellulose-rich material was separated by filtration. Ligninrich fraction was in solvent phase and used for LPU production after centrifugation to separate the aqueous and solvent phases. Vacuum evaporation was performed for lignin-rich solution to eliminate the trace amount of water.

\section{Rigid LPU synthesis from SCB organosolv lignin and Kraft lignin}

LPU synthesis from SCB organosolv lignin was performed using one-shot method by mixing all components in Table 1 [6] when silicone oil (SO) was used as chain extender. To prepare liquefied Kraft lignin, ratios of lignin to polyols were according to Table 2. Liquefaction was done in a microwave $(800 \mathrm{~W})$ for $3 \mathrm{~min}$. LPU synthesis from liquefied Kraft lignin was produced by formulations in Table 2 when silicone oil and polypropylene glycol (PPG) was used as chain extender. LPU samples were analyses for mechanical and physical properties after curing at room temperature for $48 \mathrm{~h}$.

Table 1. Formulations (g) used for synthesis of LPUs from SCB organosolv lignin

\begin{tabular}{lcccc}
\hline Components & Ethanolamine & Ethylene glycol & Amyl alcohol & Butanol \\
\hline Black liquor from SCB fractionation & 17.0 & 17.0 & 17.0 & 17.0 \\
silicone oil (SO) & 27.5 & 28.7 & 27.6 & 27.6 \\
water & 0.82 & 0.83 & 0.84 & 0.82 \\
DABCO33LV(gelling agent) & 0.71 & 0.74 & 0.68 & 0.67 \\
DABCOBL11 (blowing agent) & 0.67 & 0.68 & 0.68 & 0.70 \\
Polysiloxane (surfactant) & 0.48 & 0.49 & 0.50 & 0.46 \\
Isocyanate & 36.8 & 36.8 & 36.8 & 36.8 \\
\hline
\end{tabular}

Table 2. Formulations ( $\mathrm{g}$ ) used for synthesis of LPUs from liquefied Kraft lignin

\begin{tabular}{lllll}
\hline Components & I-PPG-A & I-PPG-B & I-SO-A & I-SO-B \\
\hline Lignin/glycerol/PEG400 & $1 / 0.4 / 2$ & $1 / 0.2 / 1$ & $1 / 0.4 / 2$ & $1 / 0.2 / 1$ \\
Liquefied Kraft lignin & 17.217 & 23.13 & 17.00 & 23.04 \\
OH group (mmol/g liquefied lignin) & 7.30 & 7.56 & 7.28 & 7.02 \\
PPG 1000 & 27.82 & 23.63 & - & - \\
Silicone oil (SO) & - & - & 27.69 & 23.55 \\
water & 0.81 & 0.83 & 0.82 & 0.82 \\
DABCO33LV(gelling agent) & 0.63 & 0.61 & 0.68 & 0.61 \\
DABCOBL11 (blowing agent) & 0.63 & 0.62 & 0.68 & 0.67 \\
Polysiloxane (surfactant) & 0.35 & 0.47 & 0.45 & 0.49 \\
Isocyanate & 36.02 & 36.00 & 36.81 & 36.14 \\
\hline
\end{tabular}

$\mathrm{OH}$ groups in black liquor from SCB fractionation and liquefied Kraft lignin were quantified by titration (ASTM D284969). $1 \mathrm{~g}$ liquefied lignin and $25 \mathrm{ml}$ of phthalate reagent were heated at $110^{\circ} \mathrm{C}$ for $20 \mathrm{~min}$. Subsequently, $50 \mathrm{ml}$ of pure 1.4-dioxane and $25 \mathrm{ml}$ of distilled water were added. The mixture was titrated with a $1 \mathrm{M}$ sodium hydroxide solution to the equivalence point. The hydroxyl number in $\mathrm{mmol} \mathrm{OH} / \mathrm{g}$ liquefied lignin was calculated by the following equation: Hydroxyl number $(\mathrm{OH})=\left[(\mathrm{B}-\mathrm{A})^{*} \mathrm{~N}\right] / \mathrm{W}$, where $A$ is the volume of the sodium hydroxide solution 
required for titration of liquefied lignin sample $(\mathrm{ml}), B$ is the volume of blank solution $(\mathrm{ml}), N$ is the normality of the sodium hydroxide solution, and $W$ is the weight of liquefied lignin (g) [6].

UV-vis spectroscopic analysis was performed to quantify the amount of SCB lignin released into black liquor during fractionation and hydrolysis reaction at the wave length of $280 \mathrm{~nm}$ when alkali lignin (Sigma-aldrich.com) was used as standard. FTIR-ATR spectra of SCB organosolv lignin and LPU sample were measured from $400-4000 \mathrm{~cm}^{-1}$ using $4 \mathrm{~cm}^{-1}$ resolution with 128 scan numbers (PERKIN ELMER Spectrum 2000). Thermogravimetric analysis (TGA) was carried out under a nitrogen gas atmosphere. The samples were heated from room temperature to $900{ }^{\circ} \mathrm{C}$ at a rate of 10 ${ }^{\circ} \mathrm{C} / \mathrm{min}$. SEM analysis (Jeol JSM5410LV) was used to study the microstructure of the LPU. Gold was coated on LPU sample prior to measurement. Mechanical properties of LPU sample was measured according to ASTM D1621 (Instron 4467) using with $2 \mathrm{~mm} / \mathrm{min}$ cross head speed and 0.5 $\mathrm{kN}$ load.

\section{Results and Discussion}

SCB organosolv lignin fractionation and hydrolysis

Amount of lignin released into black liquor from different organosolv systems was analyzed by absorbance of UV-vis spectroscopy at $280 \mathrm{~nm}$. Lignin fractionation catalyzed by $\mathrm{NaOH}$ catalyst in aqueous organosolv system (Fig. 2(A)) released significantly higher amount of lignin into black liquor compared with the control system without $\mathrm{NaOH}$ catalyst (Fig. 2(B)). Owing to high amount of lignin released, the $\mathrm{NaOH} /$ organosolv systems that were suitable for SCB lignin fractionation consisted of amyl alcohol, butanol, ethanolamine and ethylene glycol, respectively. Apart from that, these organosolv systems showed two phases of lignin-rich fraction separated from aqueous phase, and thus lignin fraction was easily collected. Although, ethanol showed high absorbance of lignin vibration, the lignin fraction in black liquor was difficultly separated, thus the ethanol organosolv system was not selected for LPU synthesis in the present study.
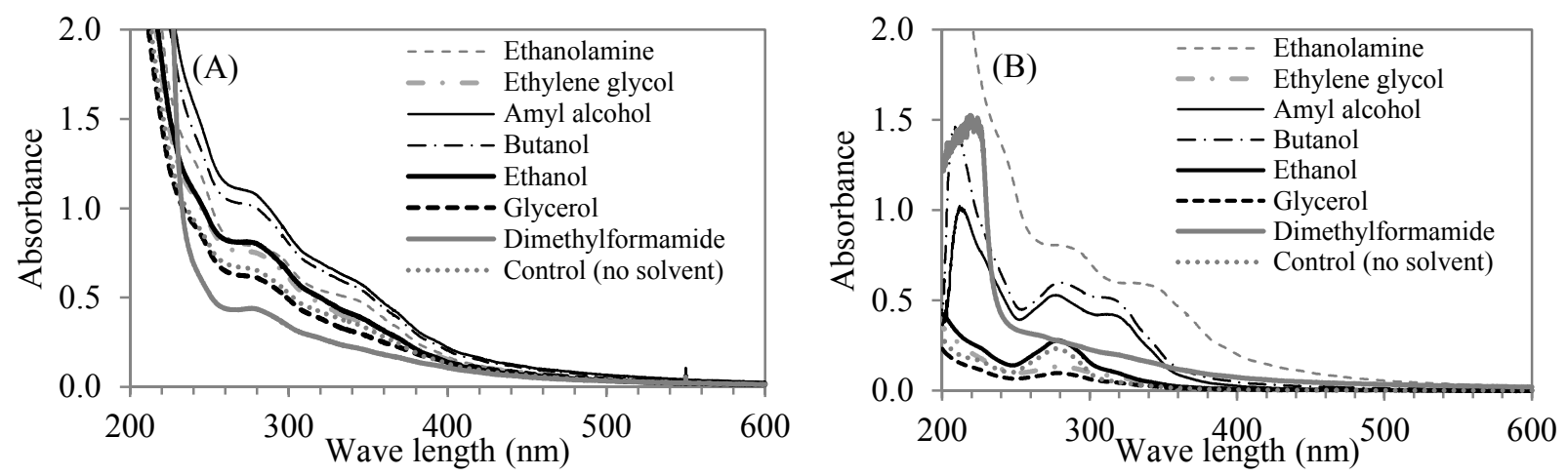

Fig. $2 \mathrm{UV}$-vis spectroscopy of lignin released into black liquor (A) in the presence of $\mathrm{NaOH}$ catalyst and (B) the control (without catalyst) in aqueous organosolv system at $170{ }^{\circ} \mathrm{C}$ for $3 \mathrm{~h}$

Regarding the degree of lignin hydrolysis, an increase of $\mathrm{OH}$ group suggested that lignin was modified to lignin polyols that can be used as monomer for LPU formation. $\mathrm{NaOH}$ catalyst showed better performance on increasing $\mathrm{OH}$ groups in lignin molecules especially when using ethanolamine as solvent compared with the control (Fig. 3(A)). This was confirmed by FTIR spectroscopy (Fig. 3(B)) for lignin derived from $\mathrm{NaOH} /$ ethanolamine organosolv system that had highest $\mathrm{OH}$-to-aromatic lignin ratio of transmittance intensity near $3400 \mathrm{~cm}^{-1}$ and $1600 \mathrm{~cm}^{-1}$ (Fig. 3(C)), respectively. These $\mathrm{OH}$ groups apparently facilitated to form urethane linkages with cyanate group during LPU synthesis.

Among all alcohols investigated, ethanolamine achieved the highest degree of lignin hydrolysis by increasing $\mathrm{OH}$ groups of black liquor. It has been reported that high holocellulose content and alpha-cellulose content and the lowest lignin content was achieved using high ethanolamine and soda pretreatment of olive wood pulping at high temperature $\left(165-195^{\circ} \mathrm{C}\right)$ [7]. Moreover, a recent work revealed that using ethanolamine as solvent for biomass pretreatment significant increased lignin extraction since it enhanced the Hildebrand solubility parameters of the mixture solvents to 
similar level of lignin solubility. It is likely that these solvents which have similar Hildebrand solubility parameters with that of lignin could offer great dissolution and extraction of lignin [8]. After lignin solubilization, base catalyst efficiently catalyzed lignin hydrolysis by $\left[\mathrm{OH}^{-}\right]$ions [9] led to the production of a monomers-rich fraction as well as enhancement of $\mathrm{OH}$ groups of lignin polyol.
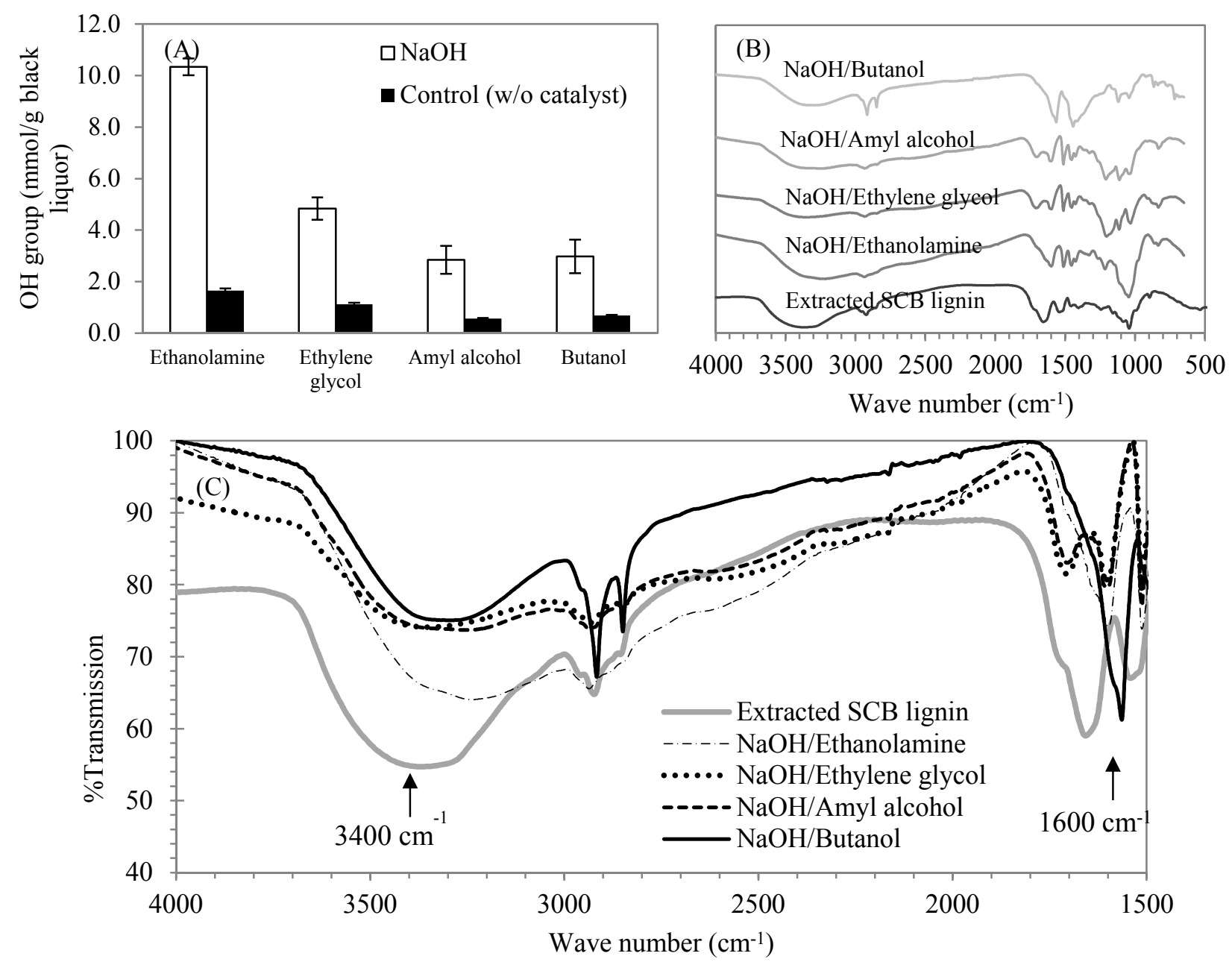

Fig. 3 (A) OH group in black liquor, (B) FTIR-ATR spectra of SCB organosolv lignin, and (C) $\%$ transmission of FTIR-ATR spectra of SCB organosolv lignin between 1500 and $4000 \mathrm{~cm}^{-1}$.

Rigid LPU synthesis from SCB organosolv lignin and Kraft lignin

Among LPU synthesis from SCB organosolv lignin, only LPU from $\mathrm{NaOH} /$ Ethanolamine was formed properly and further analyzed for mechanical properties. The others were broken when removed from the mold. Thus, the design and selection of soft material that can be used as a mold for LPU formation are very important. However, in case of chemical analysis of LPU as shown in Fig. 4(A), the FT-IR spectra of all rigid LPU samples were relatively similar where typical linkages of PU can be readily seen e.g., $\mathrm{N}-\mathrm{H}$ stretching and bending vibrations $\left(\sim 3310 \mathrm{~cm}^{-1}\right.$ and $\sim 1513 \mathrm{~cm}^{-}$ $\left.{ }^{1}\right), \quad \mathrm{OC}=\mathrm{O} \quad\left(\sim 1730 \mathrm{~cm}^{-1}\right), \quad \mathrm{CO}-\mathrm{NH} \quad\left(\sim 1640 \mathrm{~cm}^{-1}\right)$. The reason that LPU material from $\mathrm{NaOH} /$ Ethanolamine lignin had most stable shape could be explained by FTIR spectra as it contained the strongest peak intensity near $3310 \mathrm{~cm}^{-1}$ assigned to the urethane linkage (-NH stretching) and the lowest peak intensity of -NCO near $2275 \mathrm{~cm}^{-1}$ attributed to unreacted isocyanate compared to other LPUs from lignin derived from amyl alcohol, butanol and ethylene glycol organosolv system.

LPU samples were able to form from all formulations when using Kraft lignin as precursor. Similar peaks were found for LPUs from Kraft lignin and SCB organosolv lignin as shown in Fig. 4(B). However, aliphatic $\mathrm{C}-\mathrm{H}$ and $\mathrm{CH}_{3}\left(\sim 2965 \mathrm{~cm}^{-1}\right)$ vibration peak was intense for LPUs from Kraft lignin indicating poorer accessibility of lignin structure of Kraft lignin and unreacted $\mathrm{CH}$ and 
$\mathrm{CH}_{3}$ toward cyanate group compared to SCB organosolv lignin. The lowest intensity of this peak was obtained from I-SO-B sample that meant the silicone oil as chain extender could increase the accessibility of hydroxyl groups in lignin and improve the crosslink between isocyanate and lignin. This effect additionally caused improvement of compressive strength of LPU as discussed later on.
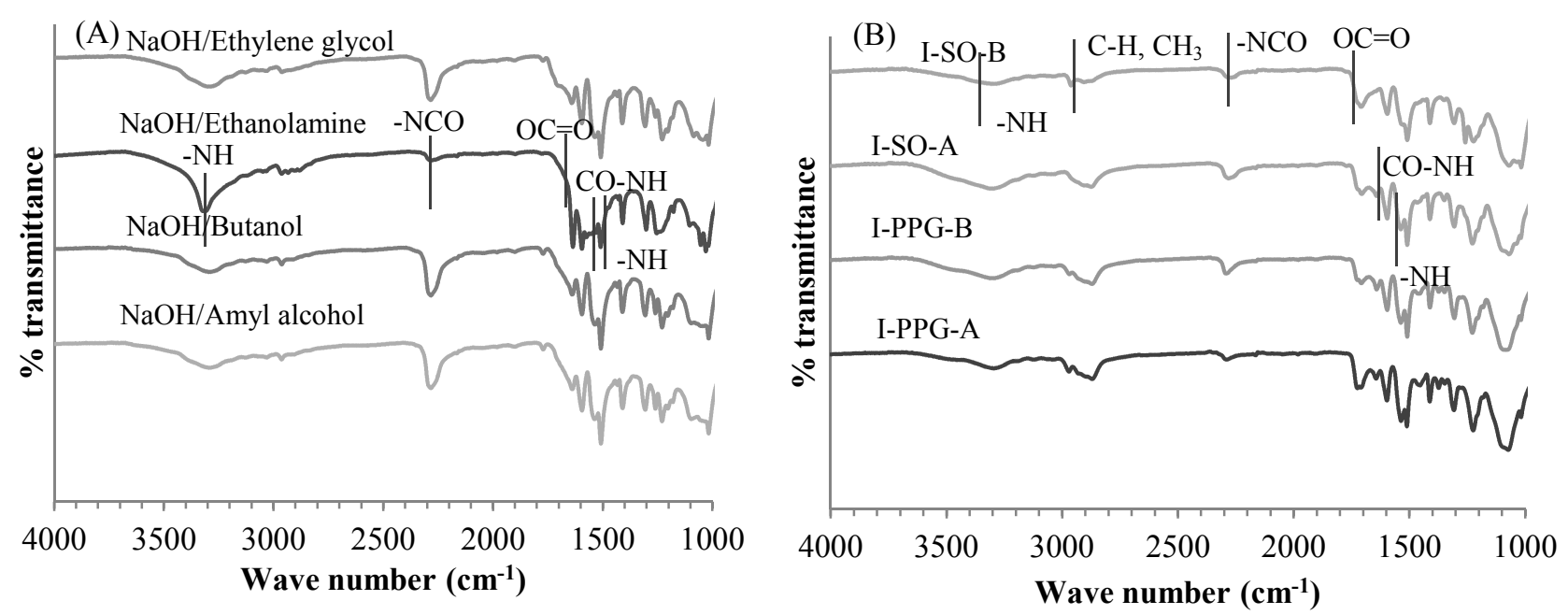

Fig. 4 FTIR-ATR spectra of LPUs from (A) SCB organosolv lignin and (B) liquefied Kraft lignin

The thermo-degradation stages of a polyurethane network based on lignin have been commonly studied through TGA (Fig. 5(A)), and it was dem onstrated that these polyurethanes show two or three degradation phases via DTG peaks (data not shown). The first degradation step was the degradation of urethane bonds at around $200{ }^{\circ} \mathrm{C}[6]$. The two degradation phases at $300-360{ }^{\circ} \mathrm{C}$ and $400-450{ }^{\circ} \mathrm{C}$ were associated with the presence of two phases within the material. The phenomenon at lower temperature is related to soft segments e.g. chain extender and at the higher temperature is related to hard segments in which the lignin is inserted. LPU from SCB organosolv lignin showed very promising TGA result as the degradation peak at highest temperature was shifted to $450{ }^{\circ} \mathrm{C}$ suggesting a high thermal resistivity of material for further application as insulator.

The strength is proportional to the density and is related to the amount of lignin that because of its aromatic rings led to an increase in chain stiffness. A higher compressive stength indicates that the material is firmer resulting from either a higher crosslink density or a higher density or both. As shown in Fig. 5(B), the highest compressive strength $(1.507 \mathrm{MPa})$ was obtained from I-SO-B sample while LPU sample from $\mathrm{NaOH} /$ Ethanolamine lignin also gave good compressive strength at $1.165 \mathrm{MPa}$ with slightly higher flexibility. Mechanical property of other samples i.e. I-SO-A, IPPG-A and I-PPG-B was not desirable that was possibly caused by inappropriate proportion of $\mathrm{NCO} / \mathrm{OH}$ ratio of the formulation which is necessary to be further optimized. Chain extenders generally have low molecular weight and are bifunctional compounds for enhancing the crosslinking in polyurethane [5]. PPG was not as effective as silicone oil as chain extender, presumably because the hydroxyl group of PPG consumed more isocyanate than silicone oil, thereby reducing the crosslinking density between isocyanate and lignin and consequently the strength of the LPUs. Density of LPU samples ranged from 0.53 to $0.97 \mathrm{~g} / \mathrm{cm}^{3}$ which was rather high compared to commercial rigid LPU foam used for insulation $\left(0.12 \mathrm{~g} / \mathrm{cm}^{3}\right)$. This was likely because lignin affected the uniformity of the cells and part of the lignin was even not well dispersed in the material and assembled together as big granules, which reduced the void volume and increased density as shown in Fig. 6. 

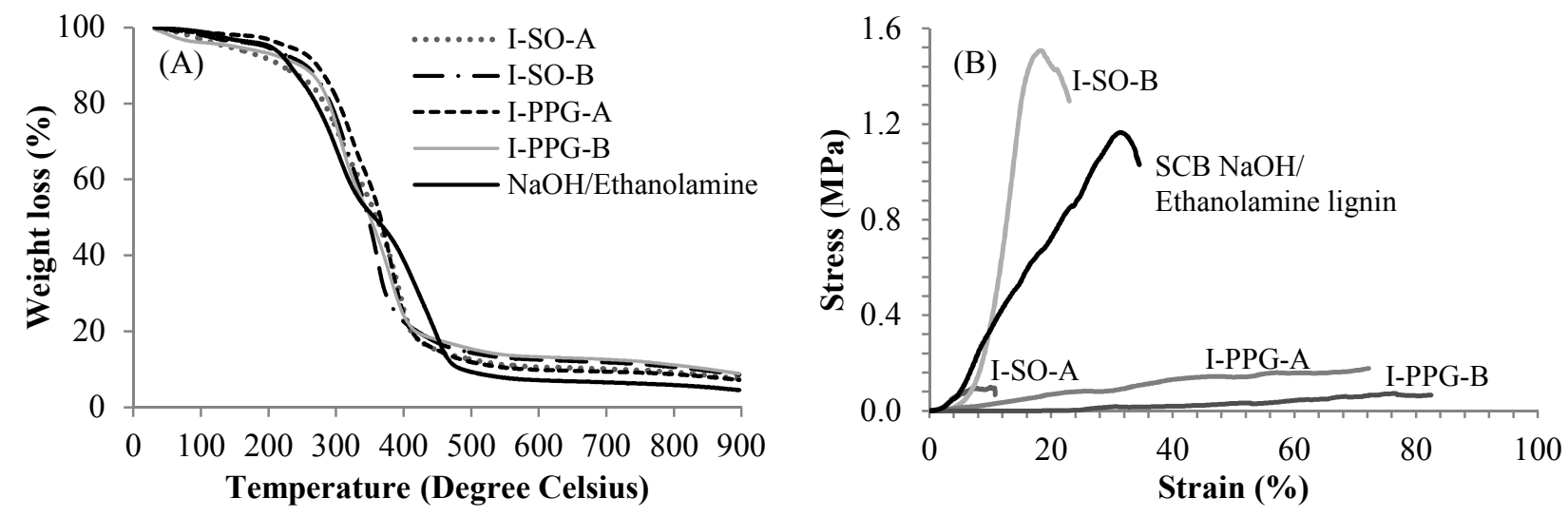

Fig. 5 (A) TGA profiles and (B) mechanical property of LPU samples from SCB organosolv lignin and liquefied Kraft lignin
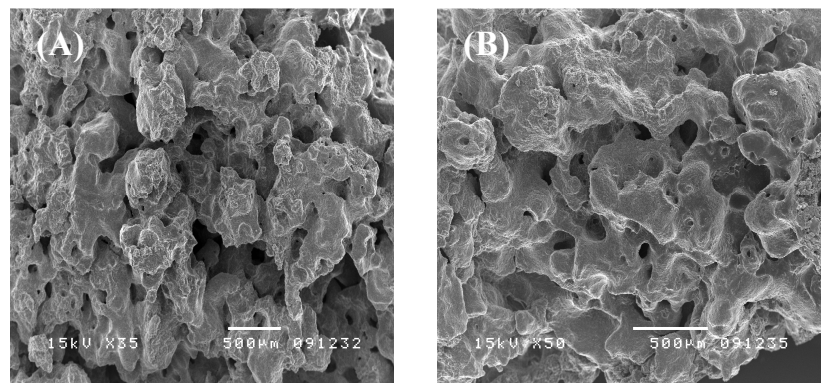

Fig. 6 SEM images of LPU from (A) Kraft lignin (I-SO-B) and (B) SCB NaOH/Ethanolaime lignin

\section{Summary}

Lignin fractionation and hydrolysis in $\mathrm{NaOH} /$ Ethanolamine system significantly increased $\mathrm{OH}$ groups of lignin polyols. LPU synthesized from SCB lignin gave comparable properties compared to Kraft lignin in terms of appearance, density and compressive strength. An optimization of LPU formulation is necessary to obtain the most preferable properties of lignin-based PU material.

\section{Acknowledgement}

This work was supported by the TRG grant (TRG 5780190) from The Thailand Research Fund (TRF) and Mahidol University.

\section{References}

[1] Y. Li, A.J. Ragauskas, Ethanol organosolv lignin-based rigid polyurethane foam reinforced with cellulose nanowhiskers, RSC Advances, 2 (2012) 3347-3351.

[2] C. Sakdaronnarong, N. Srimarut, N. Lucknakhul, N. Na-songkla, W. Jonglertjunya, Two-step acid and alkaline ethanolysis/alkaline peroxide fractionation of sugarcane bagasse and rice straw for production of polylactic acid precursor, Biochem. Eng. J., 85 (2014) 49-62.

[3] B. Gutiérrez-Rivera, B. Ortiz-Muñiz, J. Gómez-Rodríguez, A. Cárdenas-Cágal, J.M. Domínguez González, M.G. Aguilar-Uscanga, Bioethanol production from hydrolyzed sugarcane bagasse supplemented with molasses "B" in a mixed yeast culture, Renew. Energy, 74 (2015) 399-405.

[4] E. Sjöström, Wood chemistry, 2 ed., Academic Press, Inc., London, 1992.

[5] X. Pan, J.N. Saddler, Effect of replacing polyol by organosolv and kraft lignin on the property and structure of rigid polyurethane foam, Biotechnology for Biofuels, 6 (2013) 1-10.

[6] P. Cinelli, I. Anguillesi, A. Lazzeri, Green synthesis of flexible polyurethane foams from liquefied lignin, European Polymer Journal, 49 (2013) 1174-1184.

[7] L. Jiménez, A. Rodríguez, A.M. Calero, M.E. Eugenio, Use of Ethanolamine-Soda-Water Mixtures for Pulping Olive Wood Trimmings, Chem. Eng. Res. Des., 82 (2004) 1037-1042.

[8] P. Weerachanchai, S.K. Kwak, J.-M. Lee, Effects of solubility properties of solvents and biomass on biomass pretreatment, Bioresour. Technol., 170 (2014) 160-166.

[9] N. Mahmood, Z. Yuan, J. Schmidt, C. Xu, Production of polyols via direct hydrolysis of kraft lignin: Effect of process parameters, Bioresour. Technol., 139 (2013) 13-20. 